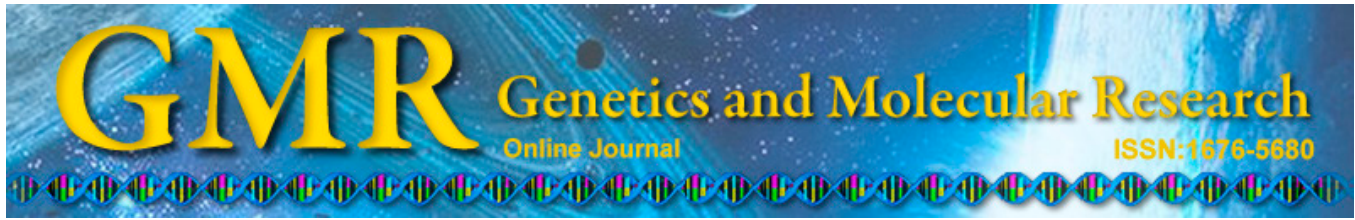

\title{
Interferon- $\alpha-2 b$ as an adjuvant therapy prolongs survival of patients with previously resected oral muscosal melanoma
}

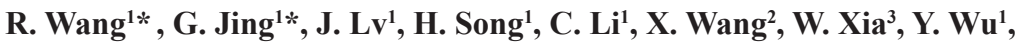 \\ G. Ren $^{1}$ and W. Guo ${ }^{1}$ \\ ${ }^{1}$ The Ninth People's Hospital Affiliated with Shanghai Jiaotong University, \\ Shanghai Key Laboratory of Stomatology, Shanghai, China \\ ${ }^{2}$ Nanjing Medical University Affiliated with Wuxi People's Hospital, \\ Wuxi City, China \\ ${ }^{3}$ The University of Texas, MD Anderson Cancer Center, Houston, TX, USA \\ *These authors contributed equally to this study. \\ Corresponding author: G. Ren \\ E-mail: renguoxin_rgx@yeah.net
}

Genet. Mol. Res. 14 (4): 11944-11954 (2015)

Received December 10, 2014

Accepted May 14, 2015

Published October 5, 2015

DOI http://dx.doi.org/10.4238/2015.October.5.8

\begin{abstract}
Two major subtypes of melanoma include cutaneous melanoma and mucosal melanoma. The latter type is rare and usually occurs in the head and neck region. High-dose interferon- $\alpha-2 b$ (IFN$\alpha-2 b$ ) has proven effective in the treatment of cutaneous melanoma. Recently, a regimen of temozolomide plus cisplatin was reported more likely to improve relapse-free survival and overall survival than high-dose IFN- $\alpha-2 b$ for mucosal melanoma. We conducted this study to analyze the therapeutic effect of high-dose IFN- $\alpha-2 b$ for patients with oral mucosal melanoma who had received prior chemotherapy. One hundred and seventeen patients with stage III-IVa oral mucosal melanoma who had received chemotherapy were analyzed. The overall survival and relapse-free survival were compared between the patients with/without high-dose IFN- $\alpha-2 b$. The results indicate that the IFN- $\alpha-$
\end{abstract}


$2 \mathrm{~b}$ treatment group had a longer relapse-free survival rate $(\mathrm{P}=0.0169)$ as compared to the control group. However, the overall survival was not significant between the two groups $(\mathrm{P}=0.096)$, except in patients in stage IVa, whose overall survival increased by 20 months $(\mathrm{P}=0.0146)$. The adverse reactions included a drug-induced influenza-like syndrome, gastrointestinal responses, myelosuppression, and hepatoxicity, which were predominantly of grade 1-2 and reversible. Thus, patients with resected oral mucosal melanoma, even those who have received chemotherapy, could benefit from the treatment of high-dose IFN- $\alpha-2 b$.

Key words: Oral mucosal melanoma; Survival; Interferon- $\alpha-2 b$; Adjuvant treatment; Chemotherapy

\section{INTRODUCTION}

Melanoma is a highly malignant tumor of melanocytes that are predominantly found in the skin, and occasionally elsewhere in the mucous membranes and uvea. In fact, the prognosis of mucosal melanoma (MM) is poor according to several large published retrospective studies (Patel et al., 2002; Temam et al., 2005; Jethanamest et al., 2011). Furthermore, the diagnosis and treatment of MM has been largely based on the guidelines for cutaneous melanoma $(\mathrm{CM})$, and clinical research in this field is relatively scarce.

Dacarbazine was the first approved chemotherapeutic agent for melanoma and has been used wildly for the treatment of melanoma for more than 30 years. Currently, the National Comprehensive Cancer Network (NCCN) guidelines recommend a comprehensive therapy for patients with advanced MM, in which high-dose interferon- $\alpha$ (HD IFN- $\alpha$ ) is recommended as an adjuvant treatment option for patients with resectable lymph node metastases and a high recurrence risk (Kirkwood et al., 1996; Hancock et al., 2004; Eggermont et al., 2005; Moreno et al., 2013). Temozolomide, which is an imidazotetrazine derivative of the alkylating agent dacarbazine, was recently used in a single-center clinical trial against MM. The subsequent study compared temozolomide plus cisplatin regimen adjuvant therapy for resected MM with HD IFN- $\alpha$ and surgery alone, and the results suggested that both the temozolomide plus cisplatin regimen and HD IFN- $\alpha$ improved relapse-free survival (RFS) and overall survival (OS) as compared to when surgery was administered alone (Lian et al., 2013). It is worth mentioning that the subjects had not received prior systemic chemotherapy in the trial. However, to improve survival, both dacarbazine and HD IFN- $\alpha$ have been used in the treatment for melanoma for a long time. Thus, the study presented here aims to examine the effect of HD IFN- $\alpha$ on the treatment of MM in those patients who have previously received chemotherapy.

\section{MATERIAL AND METHODS}

\section{Clinical data}

One hundred and twenty nine patients with primary oral MM (OMM) treated at the Department of Oral and Maxillofacial Head \& Neck Oncology of the Ninth People's Hospital affiliated to Shanghai Jiaotong University from May 2004 to November 2012 were retrospectively studied. According to the 7th edition of the American Joint Committee on Can- 
cer (AJCC) cancer staging manual, 12 of the 129 patients that presented with stage IVb-IVc OMM had received treatment that was significantly different from the other patients, and thus were excluded from this study. This left 117 patients with stage III-IVa resectable OMM for further analysis. All patients had a definite pathologic diagnosis of OMM, and received comprehensive and sequential treatment consisting of cryotherapy, followed by preoperative adjuvant chemotherapy, and surgical resection of the primary tumor not responsive to cryotherapy, adjuvant therapy, and rehabilitation therapy. Seventy-three patients who received HD IFN- $\alpha$ constituted the treatment group. Forty-four patients received no adjuvant treatment and constituted the control group. Fifteen patients did not complete the IFN treatment. This study was approved by the Institutional Review Board. All patients (or their legal representatives) had given written informed consent before treatments started.

\section{Treatment protocol}

Recombinant human IFN- $\alpha-2 b$ (Intron A, $15 \mathrm{MU} \cdot \mathrm{m}^{-2} \cdot \mathrm{day}^{-1}$ ) was injected intravenously five times a week for 4 weeks, followed by a 48 -week maintenance treatment with IFN- $\alpha$ $2 \mathrm{~b}\left(9 \mathrm{MU} \cdot \mathrm{m}^{-2} \cdot \mathrm{day}^{-1}\right)$ injected subcutaneously three times a week. According to the suggestion of the Chinese Society of Clinical Oncology Experts Committee, the dose of IFN- $\alpha-2 b$ in the first cycle was escalated from $3 \mathrm{MU}$ on day 1, $6 \mathrm{MU}$ on day 2, $9 \mathrm{MU}$ on day 3, and to $18 \mathrm{MU}$ on days 4 to 7 .

\section{Follow-up and definition}

The follow-ups were ended in January 2013. RFS was defined as the time from diagnosis to the recurrence of a tumor, and OS was defined as the time from diagnosis to death. Recurrence, as indicated by the presence of a tumor during the treatment of the primary tumor, was confirmed by clinical manifestations, CT, MRI, or PET-CT scans. The toxic and adverse reactions of this treatment were evaluated in compliance with the National Cancer Institute (NCI) CTC3.0.

\section{Statistical analysis}

Statistical analysis was conducted using the SAS version 8.02 software. The differences in clinical features between the treatment and control groups were compared by the chisquare and rank sum tests. The survival rates were calculated by the Kaplan-Meier method and compared by the log-rank test. The influence factors of survival were tested by Cox regression analysis. $\mathrm{P}<0.05$ with two-tail test was considered to be statistically significant.

\section{RESULTS}

\section{Epidemiological data and 1-, 3-, and 5-year OS rates}

Of the 117 stage-III-IVa patients retrospectively studied, 71 were males and 46 were females, which resulted in a male:female ratio of 1.54:1. The age ranged from 24 to 82 years (median was 54 years), and the follow-up time ranged from 3 to 94 months (median was 22 months). The medium OS was 43 months ( $95 \% \mathrm{CI}=36-62$ months), and the 1-, 3-, and 5-year OS rates were $94.5,58.7$, and $38.8 \%$, respectively. 


\section{Clinical features}

Before the initiation of the adjuvant treatment, the physical status scores (PS scores) for the patients were $0-2$, blood routine examination and hepatic and renal function were normal, and without any other significant diseases.There was no significant difference between the two groups in any of the clinical features, such as gender, age, location, clinical stage, primary lesion, lymph node, and PS scores $(\mathrm{P}>0.1$; Table 1$)$.

\begin{tabular}{|c|c|c|c|}
\hline Clinical features & Treatment group $(\mathrm{N}=73)$ & Control group $(\mathrm{N}=44)$ & $\mathrm{P}$ \\
\hline Gender & & & 0.7842 \\
\hline Male & 45 & 26 & \\
\hline Female & 28 & 18 & \\
\hline Age (year) & & & 0.4281 \\
\hline$\leq 50$ & 32 & 16 & \\
\hline$>50$ & 41 & 28 & \\
\hline Location & & & 0.8639 \\
\hline Gingiva & 31 & 20 & \\
\hline Palate & 32 & 20 & \\
\hline Others* & 10 & 4 & \\
\hline Clinical stage & & & 0.5521 \\
\hline Stage III & 34 & 23 & \\
\hline Stage IVa & 39 & 21 & \\
\hline Primary lesion & & & 0.1739 \\
\hline $\mathrm{T} 3$ & 37 & 28 & \\
\hline $\mathrm{T} 4 \mathrm{a}$ & 36 & 16 & \\
\hline Lymph node & & & 0.5081 \\
\hline No & 46 & 25 & \\
\hline N1 & 27 & 19 & \\
\hline PS score & & & 0.5765 \\
\hline 0 & 29 & 13 & \\
\hline 1 & 34 & 18 & \\
\hline 2 & 10 & 13 & \\
\hline $3-4$ & 0 & 0 & \\
\hline
\end{tabular}

*Including: buccal, soft palate, tongue, and lip.

\section{OS and RFS}

The difference in RFS between the treatment group and control group (i.e., 40 vs 22 months) was significant $(\mathrm{P}=0.0169)$, however, the difference in OS (i.e., 48 vs 33 months) was not significant $(\mathrm{P}=0.096$; Table 2; Figure $1 \mathrm{~A}$ and $\mathrm{B})$. Furthermore, there was no significant difference in OS (i.e., 72 vs 64 months, $\mathrm{P}=0.4236$ ) and RFS (i.e., 53 vs 34 months, $\mathrm{P}$ $=0.1960)$ between stage-III patients treated with or without HD IFN- $\alpha$. However, OS was 20 months longer (i.e., 40 vs 20 months, $\mathrm{P}=0.0146$ ) in stage IVa patients treated with HD IFN- $\alpha$.

It was observed that patients with cervical lymph node metastasis seemed to benefit more from HD IFN- $\alpha$ treatment than those patients without cervical lymph node metastasis, as the medium OS and RFS of the former was 21 months and 26 months longer than that of the latter $(\mathrm{P}<0.01$; Figure $2 \mathrm{~A}$ and $\mathrm{B})$. 
Table 2. Comparison of the OS and RFS between the treatment group and control group.

\begin{tabular}{|c|c|c|c|c|c|c|}
\hline Clinical features & No. (percentage) & Medium RFS/month & $\mathrm{P}$ & Medium OS/month & $P$ & Hazard rate (P value) \\
\hline Total & $117(100 \%)$ & 36 & & 43 & & \\
\hline HD-IFN (-) & $44(38 \%)$ & 22 & 0.0169 & 33 & 0.0960 & $0.391(0.0132)$ \\
\hline HD-IFN (+) & $73(62 \%)$ & 40 & & 48 & & \\
\hline III & $57(49 \%)$ & 53 & & 72 & & \\
\hline HD-IFN (-) & $23(20 \%)$ & 34 & 0.1960 & 64 & 0.4236 & $-(0.4236)$ \\
\hline HD-IFN (+) & $34(29 \%)$ & 53 & & 72 & & \\
\hline IVa & $60(51 \%)$ & 26 & & 31 & & \\
\hline HD-IFN (-) & $21(18 \%)$ & 10 & 0.0014 & 20 & 0.0146 & $0.430(0.0156)$ \\
\hline HD-IFN (+) & $39(33 \%)$ & 36 & & 40 & & \\
\hline IVa, N1 & $46(39 \%)$ & 28 & & 33 & & \\
\hline HD-IFN (-) & $19(16 \%)$ & 10 & 0.0043 & 20 & 0.0051 & $0.338(0.0057)$ \\
\hline HD-IFN $(+)$ & $27(27 \%)$ & 36 & & 41 & & \\
\hline
\end{tabular}

A

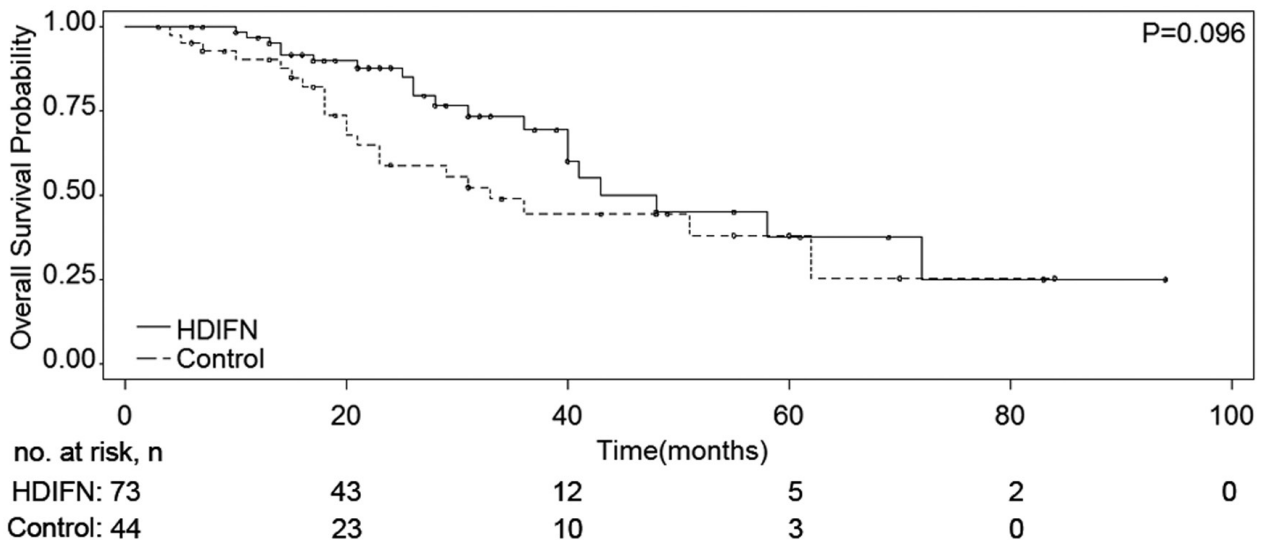

B

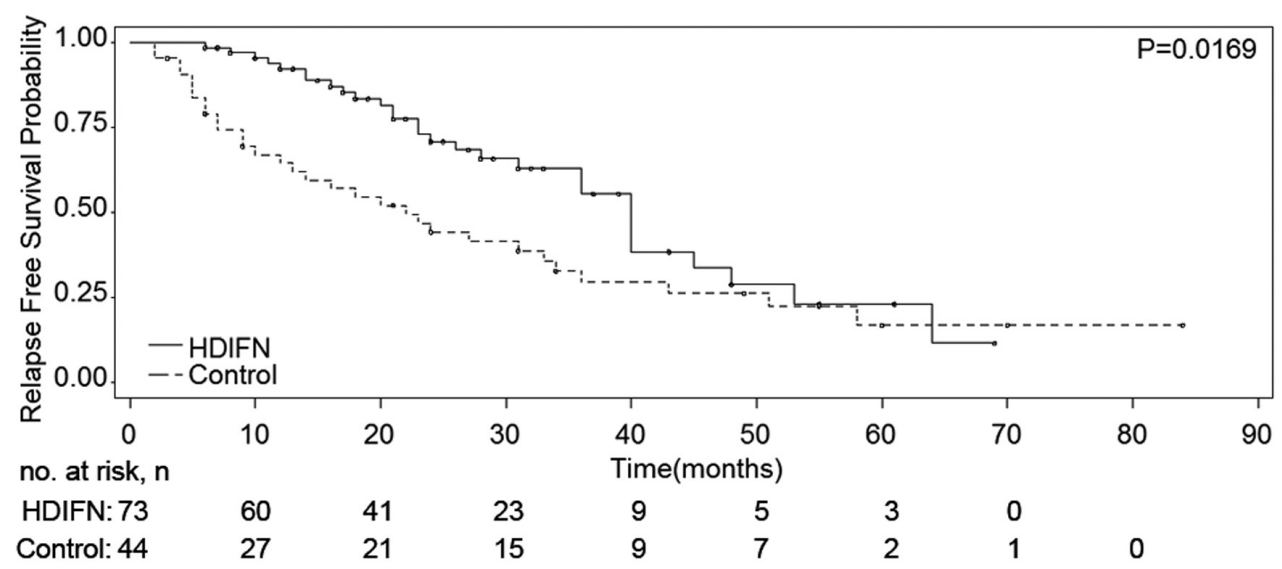

Figure 1. Kaplan-Meier curves. A. OS of the treatment group (HD IFN- $\alpha$ ) and control group. B. RFS of the treatment group (HD IFN- $\alpha$ ) and control group. 
A

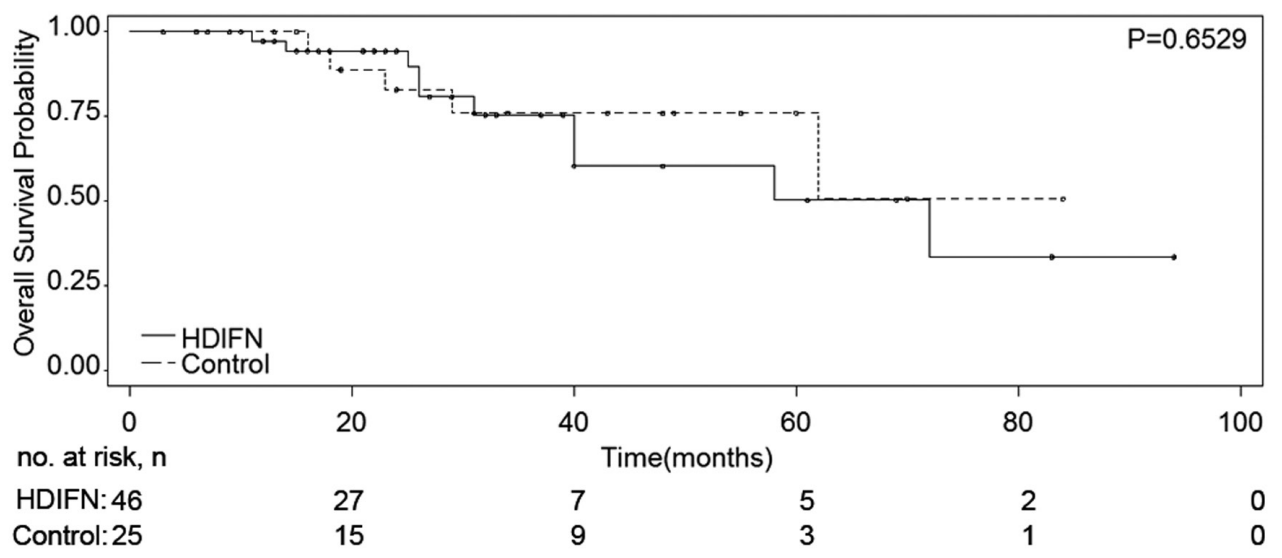

$\mathrm{B}$

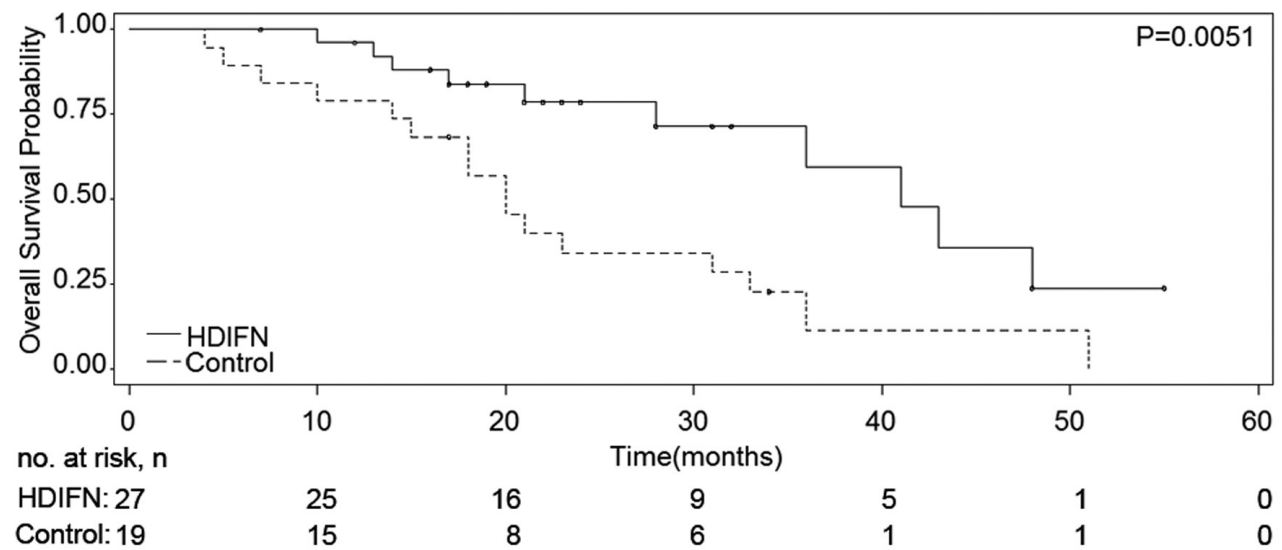

Figure 2. Kaplan-Meier curves for OS of the patients treated with/without HD IFN- $\alpha$. A. Patients without cervical lymph node metastasis. B. Patients with cervical lymph node metastasis.

\section{Adverse reactions of HD IFN- $\alpha$ treatment}

The toxic and adverse reactions of HD IFN- $\alpha$ treatment were evaluated in compliance with the NCI CTC3.0, and the results were shown in Table 3. Bone marrow depression was the most common hematologic toxicity. It was observed that leukopenia occurred in 48 of the 73 patients (66\%), mostly grade $1-2(61 \%)$ and occasionally grade $3-4(5 \%)$, followed by grade 1-2 thrombocytopenia, which occurred in 27 patients $(37 \%)$. However, these adverse reactions could be readily managed by granulocyte colony-stimulating factor. The nonhematologic toxicities observed included drug-induced influenza-like symptoms, such as fever, headache, arthralgia, and muscular soreness, which occurred in 69 of the 73 patients (94\%), followed by mild nausea or vomiting (78\%), and hepatotoxicity (48\%). These symptoms could be relieved or eliminated by symptomatic treatment including antipyretic, anal- 
gesic, antiemetic, gastric mucosal protective agent, and hepatinica. Only 7 patients had grade 3-4 toxicity and no treatment related death occurred in either group. It is worth mentioning that for the patients that did not complete the course of treatment, none of them had grade 3-4 adverse reactions.

Table 3. Main toxic and adverse reactions of high-dose IFN- $\alpha-2 b$ treatment [N (\%)].

\begin{tabular}{lccccc}
\hline & Leucopenia & Thrombocytopenia & Influenza-like syndromes & Nausea/vomiting & Hepatotoxicity \\
\hline Grade 1 & $29(40 \%)$ & $19(26 \%)$ & $55(75 \%)$ & $40(55 \%)$ & $22(30 \%)$ \\
Grade 2 & $15(21 \%)$ & $8(11 \%)$ & $14(19 \%)$ & $17(23 \%)$ & $10(14 \%)$ \\
Grade 3 & $3(4 \%)$ & $0(0)$ & $0(0)$ & $0(0)$ & $2(3 \%)$ \\
Grade 4 & $1(1 \%)$ & $0(0)$ & $0(0)$ & $0(0)$ & $1(1 \%)$ \\
Total & $48(66 \%)$ & $27(37 \%)$ & $69(94 \%)$ & $57(78 \%)$ & $35(48 \%)$ \\
\hline
\end{tabular}

\section{DISCUSSION}

Mucosal melanoma is a more common ailment with greater malignant behavior than CM. Reportedly, MM carries substantially more chromosomal aberrations and copy-number alterations than CM (Curtin et al., 2005). Moreover, the tyrosine kinase, c-kit, is overexpressed and frequently mutated in $\mathrm{MM}$, whereas the c-kit alterations are rare in CM (Curtin et al., 2006). MM is a rare disease that represents only approximately $1-2 \%$ of all malignant melanoma and usually (55\%) occurs in the head and neck region. Of the MM of the head and neck, $48 \%$ occur in the oral cavity. The incidence of MM has been reported to vary racially and regionally, with the highest incidence being among Asians (Chang et al., 1998). OMM accounts for $0.5 \%$ of all oral malignant tumors in Europe and the United States (Hicks and Flaitz, 2000), but occurred as high as $1.5 \%$ in China (Wen et al., 2001). However, it is only in recent years that research in this field has been put in the spotlight. In the past, owing to the rarity of this disease, it has been studied only sporadically with only several thousands of patients reported in the literature. Moreover, the treatment protocol for OMM was not available in the NCCN Clinical Practice Guidelines until 2010. Given these facts, this study is especially important in developing an adequate treatment of OMM.

As mentioned previously, MM is a highly malignant cancer and often carries a poor prognosis. Indeed, the reported 5-year survival rates for MM ranged from 20-35\% (Kirkwood et al., 1996; Hancock et al., 2004). Local control of a tumor seemed not to be a strong predictor of the survival of melanoma patients (Hancock et al., 2004), whereas the comprehensive treatment consisting of local surgical treatment, systemic biological treatment, chemotherapy, and targeted therapy allowed for an overall clinical benefit for these patients (Morton et al., 1999; Bedikian et al., 2008; Shiga et al., 2012; Min et al., 2014). However, despite all these improvements and ongoing research, the prognosis of MM is still poor and an effective treatment remains elusive. Unlike CM, no consensus exists regarding the standard treatment protocol for MM, most likely due to the low morbidity of this disease, which makes it difficult to conduct large-scale clinical trials and rigorous evidence-based studies.

A number of prospective clinical studies have shown a significant prolongation of both RFS and OS in early-stage patients with resectable lymph node metastases and high recurrence risk, treated with HD IFN- $\alpha$. It should be noted that no clinical benefits resulted from low- or medium-doses of IFN- $\alpha-2 b$ (Curtin et al., 2005; Eggermont et al., 2005; Moreno et al., 2013; Lian et al., 2013). In this respect, the NCCN has regarded HD IFN- $\alpha$ as the standard ad- 
juvant treatment for CM following surgery. We found that HD IFN- $\alpha$ treatment improved OS and significantly improved RFS of patients. The median RFS increased from 22 to 40 months $(\mathrm{P}=0.0169)$, and the 5-year survival rate increased to $38.8 \%$. However, stage-III patients did not benefit from the adjuvant treatment, which is likely due to the severity of the disease before treatment. In order to assess the factors that would influence survival including age, gender, location of the primary lesion, stage, and the treatment of HD IFN- $\alpha$, a Cox regression analysis was conducted. Three variables met the 0.05 level for entry into this model and became the factors affecting prognosis: stage, HD IFN- $\alpha$, and location of lesion. The hazard ratios (and P values) for the three factors above were $1.06(<0.0001), 0.391(0.0132), 1.687$ (0.0186), respectively. While stage and the location of OMM might be two risk factors affecting the prognosis, the treatment of HD IFN- $\alpha$ might be a protective factor. The clinical stage of OMM was identified as one of the most important predictors of survival for OMM patients (Figure 3), which is consistent with a previous report (Hancock et al., 2004). It is known that stage-III melanoma only involves mucosal membranes and is of mild severity and is associated with a good prognosis, while stage-IVb-IVc melanoma is found in a wide range of tissues and typically have a poor prognosis. Lesions at these stages are generally unresectable, and thus, were excluded from this study.

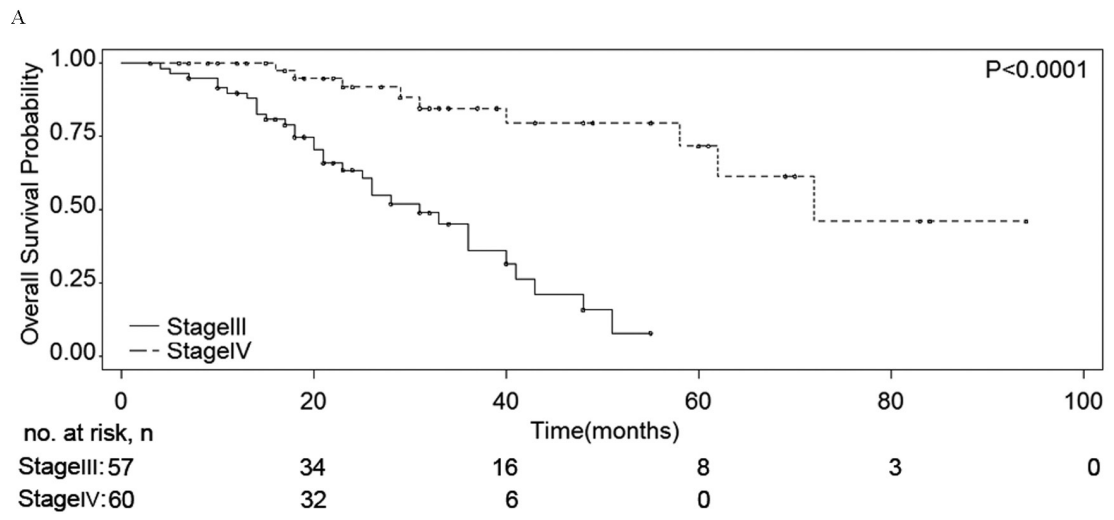

B

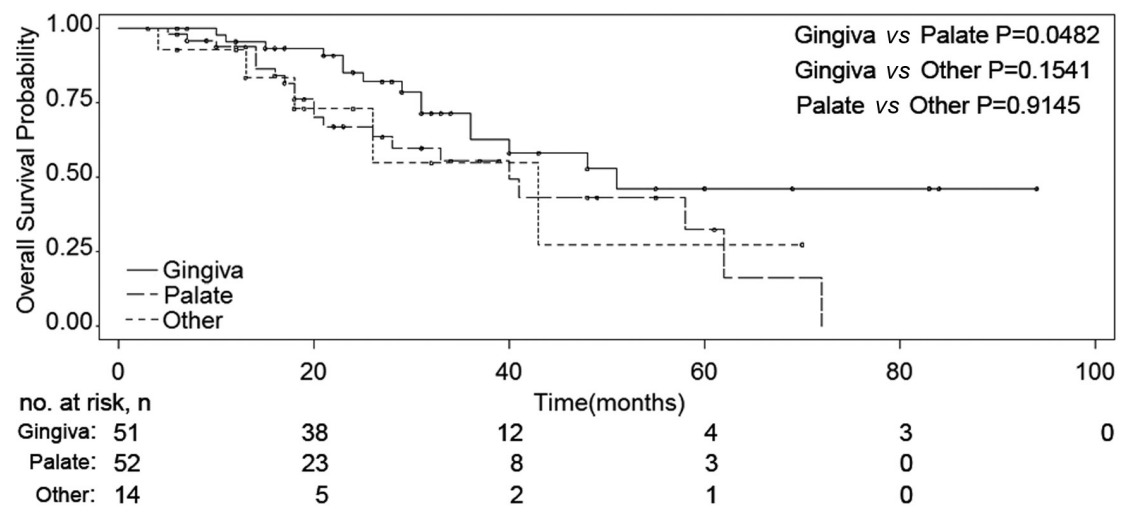

Figure 3. Kaplan-Meier curves. A. OS of the patients at stage III and stage IVa. B. OS of patients with different primary lesions. 
Another stratified analysis revealed a significant prolongation of both mediam OS and RFS in patients at stage IVa and in patients with cervical lymph node metastasis. However, according to the 7th edition of the AJCC cancer staging manual, stage I and II do not exist, and the classifications of lymph node metastases are not detailed. These might lead to confusion and bias.

The location of the primary lesions in this study included the gingiva, hard palate, buccal, soft palate, tongue, and lip. For 51 of the 117 patients, the lesions originated from gingiva, 52 patients from hard palate, and 14 patients from the other anatomical sites. The mediam OS of these groups of patients are 51 months, 40 months, and 43 months $(\mathrm{P}=0.1140)$. It seems that OMM originating from different anatomical sites have different prognoses, and those from gingiva were better off than those with hard palate OMM $(\mathrm{P}=0.0482)$, as shown in Figure 3. This difference may be due to the lesions in the gingiva being easier to be found and resected. It is worth mentioning that Lian et al. (2013) conducted a single-center clinical trial that compared temozolomide-based chemotherapy and HD IFN- $\alpha$ therapy with surgery alone, for resected MM (Curtin et al., 2006). The results suggest that the temozolomide-based chemotherapy improved RFS and OS more than HD IFN- $\alpha$. In this trial, patient lesions originated from head and neck, anorectum, and genitourinary, while it originated merely in the oral cavity in our study. Moreover, the subjects had not received prior systemic adjuvant therapy or regional radiotherapy in the trial. For those patients who have received chemotherapy, which is typically dacarbazine-based, we are unsure if the 6 cycles of temozolomide-based chemotherapy lasting 18 weeks is available, considering the drug resistance, toxicity, and side effects.

A potential difficulty with this adjuvant treatment resides in the necessity of a high dosing and a long course of treatment. Inevitably, this treatment will lead to a variety of drugrelated adverse reactions and negative social and psychological effects that may cause the patients to reduce the dosage, and in some cases, delay or even discontinue the treatment. Clearly, for successful implementation of this treatment protocol it is important to prevent or alleviate the adverse reactions and improve patient quality of life. It was reported that approximately $50 \%$ of patients that received intravenous injections and $48 \%$ that received subcutaneous injections delayed the treatment or reduced the dosage. However, the majority of patients tolerated the treatment very well and about $74 \%$ of patients continued the treatment until 1 year (or recurrence) with appropriate dose modification (Kirkwood et al., 1996). Cole et al. (1996) found that for patients with high risk melanoma, the clinical benefits of HD IFN- $\alpha$ could offset the toxic effects of the drug, and the optimal treatment protocol depended critically on the patient's tumor burden and preferences regarding toxicity and disease relapse. Of the 73 patients treated with HD IFN- $\alpha$ in our study, the majority (97\%) had minor and controllable adverse reactions, and only a small number of patients (9.5\%) had grade 3-4 adverse reactions, which were completely reversible by symptomatic treatment. It was also found that about 20 and $16 \%$ of patients delayed treatment or reduced the dosage during intravenous injection and maintenance treatment, respectively. Seventy nine percent of patients continued the treatment for at least 1 year, and $20.5 \%$ of patients did not complete the course of treatment. This constituted the main challenge for HD IFN- $\alpha$ treatment. The reasons behind patient withdrawal may be complex, and the toxic and adverse reactions might not be the decisive one. In general, the adverse reactions of HD IFN- $\alpha$ were tolerable and controllable in this study. Some rare adverse effects on the autoimmune system, such as vitiligo, or on the nervous system, such as depression and insomnia, were indeed observed during the treatment. However, abnormal thyroid function observed in previous studies (Hsieh et al., 2000, Jamil et al., 2009) was not observed in this study. The causes for these adverse reactions and late toxicity need to be elu- 
cidated in further studies. As a retrospective clinical study, it may lead to biases because of the shortage of enough randomization and perfect match. However, dacarbazine and HD IFN- $\alpha$ have proved effective for melanoma for approximately 20 years, and both have been wildly used, even together, to improve survival. Our study to some extent proved that patients could benefit from treatment with HD IFN- $\alpha$, even after they had undergone chemotherapy. In addition, if patients were diagnosed with MM and the treatment of temozolomide plus cisplatin was unavailable, the treatment presented here may provide an alternative.

In summary, as an adjuvant therapy, HD IFN- $\alpha$ can significantly improve RFS and OS of patients with primary OMM at the level of 0.05 and 0.1 , respectively. This is especially true for those with resected lymph node metastases and a high recurrence risk even though they have received dacarbazine-based chemotheraphy. Importantly, many of the adverse reactions can be controlled simply by symptomatic treatment. Thus, for those patients with high-risk MM, to whom chemotheraphy is unavailable, HD IFN- $\alpha$ may represent the treatment of choice.

\section{Conflicts of Interest}

The authors declare no conflict of interest.

\section{ACKNOWLEDGMENTS}

Research supported by the project of Science and Technology Commission of Shanghai Municipality (Grant \#12140902200) and the Medicine-Engineering Cross Funds of Shanghai Jiaotong University (Grant \#YG2012MS50).

\section{REFERENCES}

Bedikian AY, Johnson MM, Warneke CL, Papadopoulos NE, et al. (2008). Prognostic factors that determine the long-term survival of patients with unresectable metastatic melanoma. Cancer Invest. 26: 624-633.

Chang AE, Karnell LH and Menck HR (1998). The National Cancer Data Base report on cutaneous and noncutaneous melanoma: a summary of 84,836 cases from the past decade. The American College of Surgeons Commission on Cancer and the American Cancer Society. Cancer 83: 1664-1678.

Cole BF, Gelber RD, Kirkwood JM, Goldhirsch A, et al. (1996). Quality-of-life-adjusted survival analysis of interferon alfa-2b adjuvant treatment of high-risk resected cutaneous melanoma: an Eastern Cooperative Oncology Group study. J. Clin. Oncol. 14: 2666-2673.

Curtin JA, Fridlyand J, Kageshita T, Patel HN, et al. (2005). Distinct sets of genetic alterations in melanoma. N. Engl. J. Med. 353: 2135-2147.

Curtin JA, Busam K, Pinkel D and Bastian BC (2006). Somatic activation of KIT in distinct subtypes of melanoma. $J$. Clin. Oncol. 24: 4340-4346.

Eggermont AM, Suciu S, MacKie R, Ruka W, et al. (2005). EORTC Melanoma Group. Post-surgery adjuvant therapy with intermediate doses of interferon alfa $2 \mathrm{~b}$ versus observation in patients with stage IIb/III melanoma (EORTC 18952): randomised controlled trial. Lancet 366: 1189-1196.

Hancock BW, Wheatley K, Harris S, Ives N, et al. (2004). Adjuvant interferon in high-risk melanoma: the AIM HIGH Study - United Kingdom Coordinating Committee on Cancer Research randomized study of adjuvant low-dose extended-duration interferon Alfa-2a in high-risk resected malignant melanoma. J. Clin. Oncol. 22: 53-61.

Hicks MJ and Flaitz CM (2000). Oral mucosal melanoma: epidemiology and pathobiology. Oral Oncol. 36: 152-169.

Hsieh MC, Yu ML, Chuang WL, Shin SJ, et al. (2000). Virologic factors related to interferon-alpha-induced thyroid dysfunction in patients with chronic hepatitis C. Eur. J. Endocrinol. 142: 431-437.

Jamil KM, Leedman PJ, Kontorinis N, Tarquinio L, et al. (2009). Interferon-induced thyroid dysfunction in chronic hepatitis C. J. Gastroenterol. Hepatol. 24: 1017-1023.

Jethanamest D, Vila PM, Sikora AG and Morris LG (2011). Predictors of survival in mucosal melanoma of the head and 
neck. Ann. Surg. Oncol. 18: 2748-2756.

Kirkwood JM, Strawderman MH, Ernstoff MS, Smith TJ, et al. (1996). Interferon alfa-2b adjuvant therapy of high-risk resected cutaneous melanoma: the Eastern Cooperative Oncology Group Trial EST 1684. J. Clin. Oncol. 14: 7-17.

Lian B, Si L, Cui C, Chi Z, et al. (2013). Phase II randomized trial comparing high-dose IFN- $\alpha 2 b$ with temozolomide plus cisplatin as systemic adjuvant therapy for resected mucosal melanoma. Clin. Cancer Res. 19: 4488-4498.

Min KJ, Kim YS, Hong JH, Lee JK, et al. (2014). Primary malignant melanoma of uterine cervix: a suggestion of new scheme of treatment combination. Chin. J. Cancer Res. 26: 351-4.

Moreno NJ, Valero AM and Pérez TR (2013). Adjuvant treatment of melanoma. ISRN Dermatol. 2013: 1-17.

Morton DL, Ollila DW, Hsueh EC, Essner EC, et al. (1999). Cytoreductive surgery and adjuvant immunotherapy: a new management paradigm for metastatic melanoma. CA Cancer J. Clin, 49: 101-106.

Patel SG, Prasad ML, Escrig M, Singh B, et al. (2002). Primary mucosal malignant melanoma of the head and neck. Head Neck 24: 247-257.

Shiga K, Ogawa T, Kobayashi T, Ueda S, et al. (2012). Malignant melanoma of the head and neck: a multi-institutional retrospective analysis of cases in northern Japan. Head Neck 34: 1537-1541.

Temam S, Mamelle G, Marandas P, Wibault P, et al. (2005). Postoperative radiotherapy for primary mucosal melanoma of the head and neck. Cancer 103: 313-319.

Wen Y, Dai X, Wang C, Li L, et al. (2001). A retrospective clinical study of 6539 cases of malignant oral-maxillofacial tumors. Hua Xi Kou Qiang Yi Xue Za Zhi 19: 296-9. 